PROCEEDINGS OF THE

AMERICAN MATHEMATICAL SOCIETY

Volume 127, Number 7, Pages 1927-1933

S 0002-9939(99)04833-9

Article electronically published on February 11, 1999

\title{
ON SUMS AND PRODUCTS OF INTEGERS
}

\author{
YONG-GAO CHEN \\ (Communicated by David E. Rohrlich)
}

\begin{abstract}
Erdös and Szemerédi proved that if $A$ is a set of $k$ positive integers, then there must be at least $c k^{1+\delta}$ integers that can be written as the sum or product of two elements of $A$, where $c$ is a constant and $\delta>0$. Nathanson proved that the result holds for $\delta=\frac{1}{31}$. In this paper it is proved that the result holds for $\delta=\frac{1}{5}$ and $c=\frac{1}{20}$.
\end{abstract}

\section{INTRODUCTION}

Let $h \geq 2$, and let $A$ be a finite set of positive integers. Let

$$
\begin{gathered}
h A=\left\{a_{1}+a_{2}+\cdots+a_{h} \mid a_{i} \in A \text { for } i=1, \ldots, h\right\}, \\
A^{h}=\left\{a_{1} a_{2} \cdots a_{h} \mid a_{i} \in A \text { for } i=1, \ldots, h\right\} .
\end{gathered}
$$

We let

$$
E_{h}(A)=h A \cup A^{h} .
$$

It is not difficult to see that (see [3])

$$
\left|E_{h}(A)\right| \leq \frac{2}{h !} k^{h}+O\left(k^{h-1}\right) .
$$

Erdös and Szemerédi $[1,2]$ conjectured that for every $\varepsilon>0$,

$$
\left|E_{h}(A)\right| \gg k^{h-\varepsilon} \text {. }
$$

For $h=2$, Nathanson and Tenenbaum [4] have proved that if $|A|=k$ and $|2 A| \leq 3 k-4$, then

$$
\left|A^{2}\right| \gg k^{2-\varepsilon}
$$

Erdös and Szemerédi [2] showed that there exists a real number $\delta>0$ such that

$$
\left|E_{2}(A)\right| \gg|A|^{1+\delta} .
$$

Recently Nathanson [3] proved that the result holds for $\delta=\frac{1}{31}$. In this paper I show that the result holds for $\delta=\frac{1}{5}$.

Received by the editors September 24, 1997.

1991 Mathematics Subject Classification. Primary 11B05, 11B13, 11B75, 11P99, 05A17.

Key words and phrases. Additive number theory, sumsets, sums and products of integers.

This research was supported by the Fok Ying Tung Education Foundation and the National Natural Science Foundation of China. 


\section{The MAIN RESUlT}

Lemma 1. Let $a_{i}(i \in I)$ be nonnegative real numbers and $\beta>1$. Then

$$
\sum_{i \in I} a_{i}^{\beta} \geq|I|^{1-\beta}\left(\sum_{i \in I} a_{i}\right)^{\beta}
$$

In particular,

$$
\sum_{i \in I} a_{i}^{2} \geq|I|^{-1}\left(\sum_{i \in I} a_{i}\right)^{2} .
$$

Proof. By the Hölder inequality we have

$$
\sum_{i \in I} a_{i} \leq\left(\sum_{i \in I} 1^{\beta /(\beta-1)}\right)^{(\beta-1) / \beta}\left(\sum_{i \in I} a_{i}^{\beta}\right)^{1 / \beta} .
$$

Then the lemma follows immediately.

Lemma 2. Let B be a nonempty, finite set of positive integers such that

$$
\max (B) \leq 2 \min (B) .
$$

Then

$$
\left|E_{2}(B)\right| \geq \frac{7}{5} \sqrt{2}\left(\frac{|B|}{5}\right)^{5 / 4}
$$

Proof. If $\min (B) \leq 4$, it is trivial, so we may assume that $\min (B)>4$. Thus

$$
B^{2} \cap(2 B)=\varnothing \text {. }
$$

Let $|B|=k$ and

$$
l=\left[\left(\frac{k}{5}\right)^{1 / 2}\right]
$$

If $|B|<12500$, then

$$
\left|E_{2}(B)\right| \geq 2(2|B|-1) \geq 3|B| \geq 2\left(\frac{|B|}{5}\right)^{5 / 4},
$$

so we may also assume that $|B| \geq 12500$. Thus $l \geq 49$. Let

$$
\begin{gathered}
B=\left\{b_{1}, b_{2}, \ldots, b_{k}\right\}, \quad b_{1}<b_{2}<\cdots<b_{k} \leq 2 b_{1}, \\
d_{i}=b_{i+l-1}-b_{i}, \quad d_{i_{0}}=\min d_{i}, \\
B^{*}=\left\{b_{i_{0}}, b_{i_{0}+1}, \ldots, b_{i_{0}+l-1}\right\}, \\
B_{i}=\left\{b_{2 i l+1}, b_{2 i l+2}, \ldots, b_{2 i l+2 l}\right\}, \\
E\left(B^{*}, B_{i}\right)=\left(B^{*}+B_{i}\right) \cup B^{*} B_{i} .
\end{gathered}
$$


If $b_{1}^{*}, b_{2}^{*} \in B^{*}$ and $j-i \geq 2 l-1$, then

$$
\begin{aligned}
b_{2}^{*}+b_{j}- & \left(b_{1}^{*}+b_{i}\right)=b_{2}^{*}-b_{1}^{*}+b_{j}-b_{i}>-d_{i_{0}}+d_{i} \geq 0, \\
b_{2}^{*} b_{j}-b_{1}^{*} b_{i} & =\left(b_{1}^{*}-\frac{1}{2} b_{j}\right)\left(b_{j}-b_{i}\right)+b_{j}\left(\frac{b_{j}-b_{i}}{2}+b_{2}^{*}-b_{1}^{*}\right) \\
> & b_{j}\left(\frac{d_{i}+d_{i+l-1}}{2}-d_{i_{0}}\right) \geq 0 .
\end{aligned}
$$

Hence, if $j-i \geq 2$, then

$$
\begin{gathered}
\left(B^{*}+B_{i}\right) \cap\left(B^{*}+B_{j}\right)=\varnothing, \\
B^{*} B_{i} \cap B^{*} B_{j}=\varnothing .
\end{gathered}
$$

Let

$$
\begin{aligned}
B(i, u, v)=\{ & \left(b_{1}^{*}, b_{2}^{*}, b_{3}^{*}, b_{4}^{*}\right) \in\left(B^{*}\right)^{4}: b_{1}^{*} \neq b_{2}^{*} \\
& \text { and there exist } b_{3}^{\prime}, b_{4}^{\prime} \in B_{i} \text { such that } \\
& \left.b_{1}^{*}+b_{3}^{\prime}=b_{2}^{*}+b_{4}^{\prime}=u, b_{3}^{*} b_{3}^{\prime}=b_{4}^{*} b_{4}^{\prime}=v\right\} .
\end{aligned}
$$

Suppose that

$$
B\left(i_{1}, u_{1}, v_{1}\right) \cap B\left(i_{2}, u_{2}, v_{2}\right) \neq \varnothing,
$$

say

$$
\left(b_{1}^{*}, b_{2}^{*}, b_{3}^{*}, b_{4}^{*}\right) \in B\left(i_{1}, u_{1}, v_{1}\right) \cap B\left(i_{2}, u_{2}, v_{2}\right) .
$$

Then

$$
b_{1}^{*}+x=b_{2}^{*}+y, \quad b_{3}^{*} x=b_{4}^{*} y
$$

has at least one solution in integers $x, y$. It is easy to see that (3) has at most one solution. So (3) has a unique solution $x_{0}, y_{0}$. Thus

$$
x_{0} \in B_{i_{1}} \cap B_{i_{2}}, \quad u_{1}=b_{1}^{*}+x_{0}=u_{2}, \quad v_{1}=b_{3}^{*} x_{0}=v_{2},
$$

whence $i_{1}=i_{2}, u_{1}=u_{2}, v_{1}=v_{2}$. Hence

$$
\sum_{i, u, v}|B(i, u, v)| \leq\left|B^{*}\right|^{4}-\left|B^{*}\right|^{3}=l^{4}-l^{3} .
$$

Let

$$
\begin{gathered}
B_{i}^{(v)}=\left\{b: b \in B_{i} \text { and there exists a } b^{*} \in B^{*} \text { such that } b^{*} b=v\right\}, \\
\rho_{i, v}(u)=\mid\left\{b^{*}: b^{*} \in B^{*} \text { and there exists a } b^{\prime} \in B_{i}^{(v)} \text { such that } b^{*}+b^{\prime}=u\right\} \mid .
\end{gathered}
$$

Then

$$
\begin{gathered}
|B(i, u, v)| \geq \rho_{i, v}(u)\left(\rho_{i, v}(u)-1\right), \\
\sum_{u \in B^{*}+B_{i}^{(v)}} \rho_{i, v}(u)=\left|B^{*}\right|\left|B_{i}^{(v)}\right|=l\left|B_{i}^{(v)}\right| .
\end{gathered}
$$


By Lemma 1 and (5) we have

$$
\begin{aligned}
\sum_{u, v} \mid & |B(i, u, v)| \geq \sum_{v \in B^{*} B_{i}} \sum_{u \in B^{*}+B_{i}^{(v)}}|B(i, u, v)| \\
& \geq \sum_{v \in B^{*} B_{i}} \sum_{u \in B^{*}+B_{i}^{(v)}} \rho_{i, v}(u)\left(\rho_{i, v}(u)-1\right) \\
& \geq \sum_{v \in B^{*} B_{i}}\left(\left|B^{*}+B_{i}^{(v)}\right|^{-1}\left(\sum_{u \in B^{*}+B_{i}^{(v)}} \rho_{i, v}(u)\right)^{2}-\sum_{u \in B^{*}+B_{i}^{(v)}} \rho_{i, v}(u)\right) \\
& \geq \sum_{v \in B^{*} B_{i}}\left(\left|B^{*}+B_{i}^{(v)}\right|^{-1} l^{2}\left|B_{i}^{(v)}\right|^{2}-l\left|B_{i}^{(v)}\right|\right) \\
& \geq l^{2}\left|B^{*}+B_{i}\right|^{-1} \sum_{v \in B^{*} B_{i}}\left|B_{i}^{(v)}\right|^{2}-l \sum_{v \in B^{*} B_{i}}\left|B_{i}^{(v)}\right| \\
& \geq l^{2}\left|B^{*}+B_{i}\right|^{-1}\left|B^{*} B_{i}\right|^{-1}\left(\sum_{v \in B^{*} B_{i}}\left|B_{i}^{(v)}\right|\right)^{2}-l \sum_{v \in B^{*} B_{i}}\left|B_{i}^{(v)}\right| \\
& \geq 4 l^{6}\left|B^{*}+B_{i}\right|^{-1}\left|B^{*} B_{i}\right|^{-1}-2 l^{3} \\
& \geq 16 l^{6}\left(\left|B^{*}+B_{i}\right|+\left|B^{*} B_{i}\right|\right)^{-2}-2 l^{3} \\
& \geq 16 l^{6}\left|E\left(B^{*}, B_{i}\right)\right|^{-2}-2 l^{3} .
\end{aligned}
$$

Let

$$
I_{1}=\left\{i:\left|E\left(B^{*}, B_{i}\right)\right|<2 l^{3 / 2}\right\} \quad \text { and } \quad I_{2}=\left\{i:\left|E\left(B^{*}, B_{i}\right)\right| \geq 2 l^{3 / 2}\right\} .
$$

If $I_{1} \neq \varnothing$, then by (4) and the inequality just above we have

$$
\begin{aligned}
2 l^{3}\left|I_{1}\right| & <\sum_{i \in I_{1}}\left(16 l^{6}\left|E\left(B^{*}, B_{i}\right)\right|^{-2}-2 l^{3}\right) \\
& \leq \sum_{i \in I_{1}} \sum_{u, v}|B(i, u, v)| \leq l^{4}-l^{3} .
\end{aligned}
$$

So

$$
\begin{aligned}
& \left|I_{1}\right|<\frac{1}{2} l-\frac{1}{2}, \quad\left|I_{1}\right| \leq \frac{1}{2} l-1, \\
& \left|I_{2}\right| \geq\left[\frac{k}{2 l}\right]-\frac{1}{2} l+1 \geq \frac{k}{2 l}-\frac{1}{2} l .
\end{aligned}
$$

By (1), (2) and $l \geq 49$ we have

$$
\begin{aligned}
\left|E_{2}(B)\right| & \geq\left|\bigcup_{i \in I_{2}} E\left(B^{*}, B_{i}\right)\right| \geq \frac{1}{2} \sum_{i \in I_{2}}\left|E\left(B^{*}, B_{i}\right)\right| \geq \frac{1}{2} \cdot 2\left|I_{2}\right| l^{3 / 2} \\
& \geq \frac{1}{2}\left(k-l^{2}\right)(l+1)^{1 / 2}\left(\frac{l}{l+1}\right)^{1 / 2} \geq \frac{1}{2}\left(k-\frac{1}{5} k\right)\left(\frac{1}{5} k\right)^{1 / 4}\left(\frac{49}{50}\right)^{1 / 2} \\
& \geq \frac{7}{5} \sqrt{2}\left(\frac{k}{5}\right)^{5 / 4} .
\end{aligned}
$$

This completes the proof of Lemma 2 . 
Theorem. Let $A$ be a nonempty, finite set of positive integers. Then

$$
\left|E_{2}(A)\right| \geq \frac{1}{20}|A|^{6 / 5}
$$

Proof. Let

$$
|A|=k, \quad c_{1}=\frac{7}{5} \sqrt{2} 5^{-5 / 4}, \quad A_{j}=\left[2^{j-1}, 2^{j}\right) \cap A .
$$

Since

$$
\left|\bigcup_{j=1}^{\infty} A_{j}\right|=k
$$

without loss of generality, we may assume that

$$
\left|\bigcup_{j=1}^{\infty} A_{2 j-1}\right| \geq \frac{1}{2} k .
$$

Let $j_{1}, j_{2}, \ldots, j_{T}$ be all positive odd integers with $A_{j_{i}} \neq \varnothing$. Let

$$
\sum_{t \leq 0}\left|A_{j_{t}}\right|=0
$$

Since

$$
\sum_{t \leq T}\left|A_{j_{t}}\right| \geq \frac{1}{2} k
$$

there exists an integer $t_{0}$ such that

$$
\sum_{t \leq t_{0}}\left|A_{j_{t}}\right| \geq \frac{1}{12} k, \quad \sum_{t \leq t_{0}-1}\left|A_{j_{t}}\right|<\frac{1}{12} k .
$$

Thus

$$
\sum_{t \geq t_{0}}\left|A_{j_{t}}\right|=\sum_{t=1}^{T}\left|A_{j_{t}}\right|-\sum_{t \leq t_{0}-1}\left|A_{j_{t}}\right| \geq \frac{5}{12} k .
$$

If $i<u$, then $\max \left(A_{j_{i}}\right)<\min \left(A_{j_{u}}\right)$, whence

$$
A_{j_{i}}^{2} \cap A_{j_{u}}^{2}=\varnothing, \quad 2 A_{j_{i}} \cap 2 A_{j_{u}}=\varnothing .
$$

Hence each integer $n$ belongs to at most two of the sets $E_{2}\left(A_{j_{i}}\right)$. Therefore, by Lemma 1,

(6)

$$
\begin{aligned}
E_{2}(A) & \geq\left|\bigcup_{t=t_{0}}^{T} E_{2}\left(A_{j_{t}}\right)\right| \geq \frac{1}{2} \sum_{t=t_{0}}^{T}\left|E_{2}\left(A_{j_{t}}\right)\right| \geq \frac{1}{2} c_{1} \sum_{t=t_{0}}^{T}\left|A_{j_{t}}\right|^{5 / 4} \\
& \geq \frac{1}{2} c_{1}\left(T-t_{0}+1\right)^{-1 / 4}\left(\sum_{t \geq t_{0}}\left|A_{j_{t}}\right|\right)^{5 / 4} \geq \frac{1}{2} c_{1}\left(T-t_{0}+1\right)^{-1 / 4}\left(\frac{5}{12} k\right)^{5 / 4} .
\end{aligned}
$$


Suppose that $i<u$ and

$$
\begin{aligned}
& a_{i}^{\prime} \in \bigcup_{t \leq i} A_{j_{t}}, \quad a_{i} \in A_{j_{i}}, \\
& a_{u}^{\prime} \in \bigcup_{t \leq u} A_{j_{t}}, \quad a_{u} \in A_{j_{u}} .
\end{aligned}
$$

Then

$$
a_{i}^{\prime}+a_{i} \leq 2^{j_{i}+1} \leq 2^{j_{i+1}-1} \leq a_{u}<a_{u}^{\prime}+a_{u}
$$

Hence

$$
\left(\bigcup_{t \leq i} A_{j_{t}}+A_{j_{i}}\right) \cap\left(\bigcup_{t \leq u} A_{j_{t}}+A_{j_{u}}\right)=\varnothing, \quad \text { if } i \neq u
$$

Therefore

$$
\begin{aligned}
E_{2}(A) & \geq\left|\bigcup_{i=1}^{T}\left(\bigcup_{t \leq i} A_{j_{t}}+A_{j_{i}}\right)\right| \geq \sum_{i=1}^{T}\left|\bigcup_{t \leq i} A_{j_{t}}+A_{j_{i}}\right| \\
& \geq \sum_{i=1}^{T}\left|\bigcup_{t \leq i} A_{j_{t}}\right|=\sum_{i=1}^{T} \sum_{t \leq i}\left|A_{j_{t}}\right| \\
& \geq\left(T-t_{0}+1\right) \sum_{t \leq t_{0}}\left|A_{j_{t}}\right| \geq \frac{1}{12}\left(T-t_{0}+1\right) k .
\end{aligned}
$$

Therefore, by (6) and (7) we have

$$
\begin{aligned}
E_{2}(A) & \geq \max \left\{\frac{1}{2} c_{1}\left(T-t_{0}+1\right)^{-1 / 4}\left(\frac{5}{12} k\right)^{5 / 4},\left(T-t_{0}+1\right) \frac{1}{12} k\right\} \\
& \geq\left(6 c_{1}\right)^{4 / 5} \frac{5}{144} k^{6 / 5} \\
& >\frac{1}{20} k^{6 / 5} .
\end{aligned}
$$

This completes the proof of the theorem.

\section{ADDED IN PROOF}

One should also refer to Elekes, Gy., Acta Arith. 81 (1997), 365-367, for a very different method and Kevin Ford, The Ramanujan J. 2 (1998), 59-66.

\section{REFERENCES}

1. P. Erdös, Problems and results on combinatorial number theory III, M. B. Nathanson editor, Number Theory Day, New York, 1976, Lecture Notes, vol. 626, Springer-Verlag, Berlin, pp. 43-72. MR 57:12442

2. P. Erdös and E. Szemerédi, On sums and products of integers, P. Erdös, L. Alpár, G. Halász, and A. Sárközy, editors, Studies in Pure Mathematics, To the Memory of Paul Turán, Birkhäuser Verlag, Basel, pp. 213-218, 1983. MR 86m:11011 
3. M. B. Nathanson, On sums and products of integers, Proc. Amer. Math. Soc. 125 (1997), 9-15. MR 97c: 11010

4. M. B. Nathanson and G. Tenenbaum, Inverse theorems and the number of sums and products (to appear).

Department of Mathematics, Nanjing Normal University, Nanjing 210097, People's Republic of China

E-mail address: ygchen@pine.njnu.edu.cn 